\title{
Schutzschicht wiederherstellen
}

\section{Phospholipid als neue Therapieoption?}

Faktoren des Schleims scheinen die Darmmukosa gegen schädigende Effekte der Darmflora zu schützen. Neue Forschungsbefunde lassen es damit realistisch erscheinen, auf dem Boden solcher Erkenntnisse eine neue Therapieform bei der Colitis ulcerosa zu etablieren.

Unsere Darmschleimhaut wird tagtäglich mit einer Heerschar von Bakterien konfrontiert und muss sich auch gegen die übliche, vermeintlich gesunde Darmflora schützen. Der Organismus nutzt dazu offenbar Phosphatidylcholin, das in Form dicht gepackter, lamellärer Strukturen im Darm vorliegt und laut Prof. Wolfgang Stremmel, Heidelberg, Übergriffe der Bakterien auf die Darmschleimhaut verhindert. Das Phospholipid wird nach seiner Herstellung aktiv ins Darmlumen sezerniert, ein Vorgang, der durch Gallensäuren stimuliert wird. Die negativ geladenen Gallensäuren halten das positiv geladene Phosphatidylcholin direkt an der Darminnenseite fest, sodass sogenannte „Surfactant Like Bodies" entstehen und damit eine den Darm praktisch auskleidende Schutzschicht. Dieser Mukosaschutz ist bei Patienten mit Colitis ulcerosa anschei-

Besuchen Sie das Cgastro
Falk Gastro
Forum 1:0 für
die Viszeralmedizin. Es findet am
Samstag, den 30. Juni 2012, in
Leverkusen statt.
Infos: www.drfalkpharma.de/
veranstaltungen

nend gestört, denn sie weisen deutlich weniger Phosphatidylcholin im Darm auf als Gesunde.

\section{Mukusbarriere stabilisieren}

Das kann nach Stremmel wahrscheinlich mit ein Grund dafür sein, dass sich chronische Entzündungen bei den Patienten manifestieren. Die neuen Erkenntnisse nähren Hoffnungen, den pathogenetisch bedeutsamen Effekt in eine neue Behandlungsmöglichkeit bei der Colitis ulcerosa ummünzen zu können. Denn durch eine orale Verabreichung von Phosphatidylcholin in entsprechender galenischer Zubereitung sollte es möglich sein, das gestörte Gleichgewicht im Darm wiederherzustellen. „So lässt sich dann wahrscheinlich auch die Mukusbarriere wieder stabilisieren“, erläuterte der Mediziner bei einem Falk Symposium in Basel.

\section{Krankheitsremission bei jedem zweiten Patienten}

Dass die Strategie dazu beitragen kann, Entzündungsreaktionen bei der Colitis ulcerosa einzudämmen, belegt eine erste randomisierte Doppelblindstudie bei 60 Patienten. Sie wurden drei Monate lang placebo-kontrolliert mit dem Phospholipid behandelt, was nach Stremmel zu einer Besserung der Krankheitsaktivität bei mehr als

\section{Mikroskopische Kolitis}

Morbus Crohn und Colitis sind keineswegs die einzigen chronisch entzündlichen Darmerkrankungen. Auch die Mikroskopische Kolitis gehört zu dieser Krankheitsentität und sollte nach Prof.Stephan Miehlke, Hamburg, die gleiche Beachtung erfahren wie die Colitis ulcerosa und der Morbus Crohn: „Denn sie ist ebenso häufig und die Patienten sind in aller Regel genauso stark in ihrer Lebensqualität beeinträchtigt". Die Crux: Anders als Morbus Crohn und Colitis ulcerosa zeigt sich die Mikroskopische Kolitis bei der herkömmlichen endoskopischen Untersuchung nicht, sondern nur im histologischen Bild. Wichtig sind bei Patienten mit wässriger Diarrhoe deshalb Stufenbiopsien. Wird die Diagnose adäquat gestellt, ist die Behandlung keine Kunst mehr: „Mit Budesonid gibt es eine gut wirksame und gut verträgliche Therapie", so Miehlke.

90\% der Patienten führte. Jeder zweite Patient erreichte durch die Behandlung mit dem Phospholipid sogar eine Krankheitsremission.

Der Gastroenterologe ist davon überzeugt, dass eine lokale Substitution von Phosphatidylcholin, bei dem keine gravierenden Nebenwirkungen zu erwarten sind, die Behandlungsmöglichkeiten bei Patienten mit aktiver Colitis ulcerosa erweitern kann. Insbesondere, so die Hoffnungen, könnten sich bei Patienten mit steroidrefraktärer und steroidabhängiger Colitis ulcerosa Kortikosteroide einsparen lassen.

(Christine Vetter)

Falk Symposium 183 “Dealing with our In-vironment: New Aspects in IBD Pathogenesis and Therapy" und Falk Workshop "Microscopic Colitis - Creating Awareness for an Underestimated Disease", 3.-5. Mai 2012 in Basel 